\title{
Hyperdynamics for entropic systems: time-space compression and pair correlation function approximation
}

\author{
Xin Zhou ${ }^{*}{ }^{1}$ Yi Jiang, ${ }^{2}$ Kurt Kremer, ${ }^{3}$ Hans Ziock, ${ }^{1}$ and Steen Rasmussen ${ }^{1}$ \\ ${ }^{1}$ Earth and Environmental Science Division, Los Alamos National Laboratory, Los Alamos, NM 87545 \\ ${ }^{2}$ Theoretical Division, Los Alamos National Laboratory, Los Alamos, NM 87545 \\ 3 Max-Planck-Institut für Polymerforschung, Ackermannweg 10, D-55128 Mainz, Germany
}

(Dated: June 26, 2018)

\begin{abstract}
We develop a generalized hyperdynamics method, which is able to simulate slow dynamics in atomistic general (both energy and entropy-dominated) systems. We show that a few functionals of the pair correlation function, involving two-body entropy, form a low-dimensional collective space, which is a good approximation that is able to distinguish stable and transitional conformations. A bias potential, which raises the energy in stable regions, is constructed on the fly. We examine the slowly nucleation processes of a Lennard-Jones gas and show that our new method can generate correct long time dynamics without a prior knowledge.
\end{abstract}

PACS numbers: PACS numbers: 05.10-a, 02.70.Ns, 82.20.Wt, 64.70.Fx

Molecular dynamics (MD) simulations are typically limited to a time scale of less than a microsecond, so many interesting slow processes in chemistry, physics, biology and materials science cannot be simulated directly. Recently, new methods, including kinetic Monte Carlo, transition path ensemble methods, minimal action/time methods and the constrained MD simulation [1, 2, 3, 4], have been developed to study the slow processes (for a review see [5]). They all require a prior knowledge of the system, which is often hard to obtain, and they can only deal with a few special processes inside a small part of the configurational space of the system.

For many systems, the interesting dynamics are governed by the infrequent, fast transitions between metastable regions; yet the systems spend most of their time in the stable regions, whose dynamics can be well described by some time-averaged properties. Hence we would coarse grain the stable configurations while keeping the needed details in the unstable regions that define the transitions. Hyperdynamics, as developed by Voter [6] is an example of such a coarse-graining method. The hyperdynamics method treats the potential wells as the stable conformational regions that are separated by the saddle regions. A bias potential is designed to lift the energy of the system in these wells, while keeping the saddle regions intact. Dynamics on the biased potential leads to accelerated evolution from one stable region to another. Based on transition state theory, the realistic escape time $t_{\text {real }}$ from the wells can be reproduced, $t_{\text {real }}=\Delta t \sum_{i} \exp \left[\beta \Delta V\left(r\left(t_{i}\right)\right)\right]$, where $\Delta t$ is the time step of $\mathrm{MD}, \Delta V(r)$ is the applied bias potential along the simulated trajectory $r(t)$. This method has been applied successfully to systems in which the relevant states correspond to deep wells in the potential energy, with dividing surfaces at the energy ridge tops separating these states [7]. It has however not been clear

*Email: xzhou@lanl.gov how to apply hyperdynamics in cases where the transitions are dominated by entropic considerations. In such cases, the potential energy alone is not enough to distinguish stable and transition regions since some conformations with similar energy might belong to stable and transition regions, respectively. A complication that occurs even when trying to apply hyperdynamics to solids with fairly clearly defined stable regions is that after applying the bias potential, the energy landscape becomes much flatter and the system can start to have entropiclike characteristics. These effects limit the improvement in the simulation rate that can be achieved by the hyperdynamics method over the direct MD approach. Thus, although there are some attempts in the literature [8, 9 ] to apply hyperdynamics to enhance conformational sampling in bio-systems, generally, accurate slow dynamics or kinetic rates can only be expected for relatively simple solids or low dimensional systems.

In this Letter, we derive a more general hyperdynamics method that can be used to access longer time scales in fluids. We present explicit conditions for applying this method. We use the pair correlation function as a reliable means of identifying the important conformations and then construct the appropriate bias potentials in a lowerdimensional space while the simulation runs. We then examine the performance of this method by looking at the slow gas-liquid transition in a system of $N$ identical Lennard-Jones particles.

We begin this process by introducing a timecompressing transformation, $d \tau=a(r) d t$, where $d \tau$ is a pseudo-time step, $d t$ is the real time step, and the local dimensionless compression factor is given by a conformational function $a(r)$, which is $\leq 1$. Thus the trajectory, $r(t)$, can be rewritten as $r(\tau)=r(\tau(t))$ in a shorter pseudo-time interval, $\tau=t \int d r D(r ; r(t) ; t) a(r)$, where $D(r ; r(t) ; t)$ is the distribution of $r(t)$ in the interval $[0, t]$. The compressed trajectory $r(\tau)$ satisfies a new equation 
of motion,

$$
\frac{d}{d \tau} \mathbf{P}_{i}=-\frac{\partial V}{\partial \mathbf{R}_{i}}+\sum_{j} \frac{\mathbf{P}_{i}}{M} \mathbf{P}_{j} \cdot \frac{\partial}{\partial \mathbf{R}_{j}} \ln a(r)
$$

where $\mathbf{P}_{i}=M d \mathbf{R}_{i} / d \tau, M$ is the transformed mass, equal to $m a^{2}(r)$, and the $m$ is the real mass of the particles. $V(r)$ is the potential energy, and $r$ is the simple donation of the position vectors $\mathbf{R}_{i}(i=1, \cdots N)$ of all the $N$ particles. At first glance it would not appear to be advantageous to directly generate $r(\tau)$ from Eq.(1) as very short time steps are necessary due to the small values of $M$. However, if we only focus on the long-time dynamics, we can use a smoother pseudo-trajectory $\mathcal{R}(\tau)$ to replace $r(\tau)$, provided that one requires the reproduced time from $\mathcal{R}(\tau)$ to be the same as that from $r(\tau)$. Thus, a sufficient condition to replace $r(\tau)$ with $\mathcal{R}(\tau)$ is that their distributions are the same.

In general, the distribution along a finite-length trajectory is not easily known. However, in the time-consuming regions (stable regions), similar conformations would be visited many times even during a finite simulation time. Thus we can assume the distribution can be approximated by $D(r ; r(t) ; t) \propto \exp (-\beta V(r))$. Many methods might be used to generate $\mathcal{R}(\tau)$ with the required distribution. One simple method is to use a realistic trajectory corresponding to the local equilibrium of a new potential $U(r)=V(r)-k_{B} T \ln a(r)$. Actually, based on the same local equilibrium supposition, if one replaces the kinetic energy term of Eq. (11) by its ensemble averaged value, $<\mathbf{P}_{i} \mathbf{P}_{j} / M>=k_{B} T \delta_{i j}$, Eq.(11) is indeed the equation of motion of the particles with smaller mass $M$ under the new potential $U(r)$. Outside of the time-consuming regions, we choose $a(r)$ as unity so that the MD time is realistic. If we select small $a(r)$ only in the potential well regions, we effectively have the hyperdynamics presented by Voter [6]. The condition for the use of compressed time is that the simulated trajectory under $U(r)$ has the equilibrium distribution in all the biased (timecompressed) regions. Simply, we can suppose that the distribution is locally given by the Boltzmann one in the conformational regions where the value of the distribution is larger than a critical value. In comparison with the transition state theory in original hyperdynamics implementation, the new approach makes it is easier to both determine the proper regions to bias and to design the appropriate bias potential.

In solids, as the number of conformations inside stable regions (potential energy wells) is often small and the distribution of long-time trajectories can reach local equilibrium, the hyperdynamics method works very well. However, in entropy-dominated systems (e.g. gas and liquids), the number of conformations (entropy) inside time-consuming regions may be huge, and thus the Boltzmann distribution may not be reached in finite MD time. In this case, we average the neighboring distribution of the trajectory, $\bar{D}(r ; \sigma)=\int_{\sigma} D(r) d r$, where $\sigma$ is the size of selected neighbors. For the smoothed distribution, in time-consuming regions, we can expect,
$\bar{D}(r ; \sigma) \propto \int_{\sigma} \exp (-\beta V(r)) d r$. Thus, by selecting smooth $a(r)$ functions, the realistic time propagator can still be reproduced from the physical trajectory of $U(r)$, provided the averaged distribution of the $U(r)$ trajectory satisfies the equation.

As an illustration, we compress the $3 N$-dimensional flat conformational space $r$ to a curved $3 N$-dimension $q$ space, $d q=\mathbf{A}(r) d r$, where $\mathbf{A}(r)=\frac{\partial q}{\partial r}$ is the Jacobian of the transformation. In $q$ space, the trajectory $q(t)=q(r(t))$ satisfies a new equation of motion with a positive symmetric mass matrix $\mathbf{M}=\mathbf{B}^{T}(r) \mathbf{m} \mathbf{B}(r)$, where $\mathbf{B}^{T}$ denotes the transpose, $\mathbf{m}$ is the real mass diagonal matrix, and $\mathbf{B}=\mathbf{A}^{-1}$. Not losing any generality, we choose a diagonal $\mathbf{M}$ by rotating the $d q$ space, and the new equation of motion is,

$$
\frac{d}{d t} \mathbf{P}=-\frac{\partial V}{\partial q}+\sum_{j} \frac{P_{j}^{2}}{2 \mathbf{M}_{j}} \frac{\partial \ln M_{j}(q)}{\partial q},
$$

where $\mathbf{P}$ and $P_{j}$ are the generalized momentum and its $j$ component, respectively, and $M_{j}$ is the $j$ diagonal element of $\mathbf{M}$. If one replaces $\frac{P_{j}^{2}}{M_{j}}$ with $k_{B} T$, Eq.(2) is the equation of motion of heavier particles $\left(\operatorname{mass} M_{j}\right.$ ) under a new potential, $W(q)=V(q)+k_{B} T \log J(q)$, where $J(q)=\operatorname{det}(\mathbf{A})$ is the determinant of the Jacobian A of the transformation. Here, $W(q)$ is indeed the free energy of system in $q$ space. Thus, by inhomogeneously compressing the conformational space, we transform the original entropy-dominated $r$ space to a energy-dominated $q$ space with effective potential $W(q)$. If the trajectory $q(t)$ of $W(q)$ corresponds to the Boltzmann distribution in some time-consuming regions, the corresponding trajectory $r(q(t))$ represents a local equilibrium in $r$ space. Thus we can bias the effective potential $W(q)$ in $q$ space to extend the MD time scale. Actually, this can be done directly in $r$ space without using the explicit transformation $\mathbf{A}(r)$.

The keys to successfully apply hyperdynamics are distinguishing the conformations and designing suitable bias potentials $\Delta V(r)$ for the entire conformation space $r$. Obviously, $\Delta V(r)$ should have the same symmetry as $V(r)$. Considering a simple case of $N$ identical particles, we rewrite the conformational vector $\left\{\mathbf{R}_{j}\right\}(j=1, \cdots, N)$ as a density field, $\hat{\rho}(\mathbf{x})=\sum_{j} \delta\left(\mathbf{x}-\mathbf{R}_{j}\right)$. Here both $\mathbf{x}$ and $\mathbf{R}$ are the normal 3-dimension spatial vectors. Since the neighboring conformations are identical in the viewpoint of slow dynamics, $\hat{\rho}(\mathbf{x})$ can be averaged to get a smooth function, $\bar{\rho}(\mathbf{x})$, by for example using a Gaussian function to replace the Dirac- $\delta$ function. If the width of the Gaussian function is small, $\bar{\rho}(\mathbf{x})$ can be used to identify different conformations. Here we used a functional space to replace the $3 N$-dimension conformation space, but actually, the physically allowed $\bar{\rho}(\mathbf{x})$ only occupies a very small part of the functional space. By neglecting multi-body correlations and directional correlations, we can approximate the density field $\bar{\rho}(\mathbf{x})$ (or conformations) by using some bin-averaged values of the radial pair correlation function $g(x)$ of the conforma- 
tions (here, $x$ donates the length of the spatial vector $\mathbf{x}$ ), $g_{i}=\frac{1}{\Delta} \int_{\Delta} g\left(x+x_{i}\right) d x$. Here $g_{i}$ is an average value of $g(x)$ in a small bin $\left(x_{i}-\Delta / 2, x_{i}+\Delta / 2\right)$. Thus, each conformation corresponds to the group of $g_{i}$ that defines a point in $g$ space. The spatial neighbors of the conformation and their symmetric companions will also correspond to the same $g$ point. If the bin size is very small, all conformations with the same $\left\{g_{i}\right\}$ are identical in the slow dynamics viewpoint and thus the bias potential of hyperdynamics can be written as a function in the low-dimension $g$ space, $\Delta V\left(\mathbf{R}^{N}\right)=f\left(\left\{g_{i}\left(\mathbf{R}^{N}\right)\right\}\right)$. To better identify conformations even when larger bin sizes are used, some important dynamics-related physical variables, such as the potential energy $V\left(\mathbf{R}^{N}\right)$, can be added into the $\left\{g_{i}\right\}$ variable group. Another important variable is the two-body entropy, $S_{2}=-2 \pi \rho \int[g(x) \ln g(x)-g(x)+1] x^{2} d x$. which forms the main part (about 90 percent) of the macroscopic excess entropy [11]. Similarly, it is also possible to use some other functional of $g(x)$ to replace some $g_{i}$. In special systems, it may be useful to add some special order parameters $O_{j}$ to take into account possible multi-body correlations and thereby decrease the needed number of $g_{i}$. Finally, we have a group (of order 10) of general collective variables denoted as $S=\left\{S^{j}\right\}$, which might involve $V, S_{2}$, some $\left\{g_{i}\right\}$ and some possible $\left\{O_{j}\right\}$, to identify conformations and form an appropriate bias potential. In general, we construct the bias potential as $\Delta V\left(S\left(\mathbf{R}^{N}\right)\right)=k_{B} T f_{+}\left(\ln D(S) / D_{c}\right)$, where, $D(S)$ is the distribution of the simulated trajectory, $D_{c}$ is the selected critical value. The function $f_{+}(z)=z$ for larger positive $z$, and smoothly approaches 0 as $z$ decreases to 0 . The designed bias potential will generate a flatter distribution in $S$ space while the biased regions are still visited often enough for the system to reach local equilibrium. The bias potential can be formed gradually. First, we generate a long non-biased MD trajectory to calculate $D(S)$ and form a (small) bias in some $S$ regions. Next a long trajectory is simulated in the biased system, which in turn generates the basis for the next bias. This process is repeated until the desired long realistic time is reached. Thus, we can gradually study dynamics at ever longer time scales, but at the expense of the details seen in fast dynamics. With the biased potential, the bias force on particles can be calculated by the chain rule of differentiation, $\Delta \mathbf{f}_{i}=-\sum_{j} \frac{\partial \Delta V}{\partial S^{j}} \frac{\partial S^{j}}{\partial \mathbf{R}_{i}}$.

We have examined the general hyperdynamics method in a simple system of $N$ identical Lennard-Jones (LJ) particles and studied the slow gas/liquid transition in the NVT ensemble. We used the truncated and shifted LJ potential with $r_{c}=2.5$, and the reduced units: $\epsilon_{l j}=1$, $\sigma_{l j}=1$ and the mass of particles $m=1$. The temperature is fixed as $T=0.613$. The velocity Verlet algorithm was used to integrate the Langevin equation of motion. Obviously, in such a system, the potential energy of transitional conformations ( liquid drops with critical size) is lower than that of the stable gas phase. Thus, some simple bias methods, for example, just lifting the energy of all lower-energy conformations [10], cannot work at all in such a system. Actually, for general entropy-important systems, using the potential alone is not enough to identify the transitional conformations and then form the bias potential. Only in some special low-dimension systems [9] where the entropy is occasionally not important, exceptions may be found.

We initially studied a gas phase with a relatively large saturation ( a number density $n=0.02$ for a system of $N=1000$ particles) for which a liquid drop forms in normal MD without any bias. The phase transition happens in a narrow time window where the potential $V$, the twobody entropy $S_{2}$ and the pair correlation function $g(x)$ are found to change drastically in the gas/liquid phase transition process. However, inside each small $S_{2}$ range, we found $g(x)$ only fluctuates slightly around its average value. This shows that the two-body entropy $S_{2}$ integrates the information of the $g(x)$. Thus, in this simple system, we use only two functionals of $g(x)$, namely $V$ and $S_{2}$, to form the collective space while designing the bias potential. By examining the $g_{i}$, which are available from our simulations, we were able to show that $V$ and $S_{2}$ can sufficiently identify transition states and stable states, at least in this simple system. Since $S_{2}$ gives the main part of the entropy, we expect that $S_{2}$ and $V$ are also leading collective variables even in more complex systems.

When we decrease the density (or saturation) of the LJ system, the lifetime $(\tau=1 / k, k$ is the transition rate) of the gas phase increases drastically. For example, $\tau \sim 10^{4}$ when the density $n=0.016$, but increases by about a factor of 20 when the density is only slightly decreased to $n=0.014$. Even in this density region, direct simulation of the gas/liquid transition is still possible. Thus, we compared the transition kinetics with and without the use of a bias. Fig.(1) shows that the distribution of the gas to liquid transition waiting time $t$ is exponential in $t$ from both non-biased and biased simulations, $\ln P(t) \propto-t / \tau$. The boost factor $\alpha$ resulting from the hyperdynamics method, which characterizes the average gain in the rate at which time advances relative to direct $\mathrm{MD}$, is about 21, as shown in the inset of Fig.(11) ( $\alpha=1$ for unbiased MD simulation). We also directly calculate $\tau$ by averaging the transition time of full simulations for a better comparison. We found that in the biased MD case, $\tau=2.02 \times 10^{5}$, in excellent agreement with the direct MD result, $\tau=2.07 \times 10^{5}$. In our current simulations, we gradually increase the bias potential until our desired transition can happen in the usual MD steps. Thus we need and can apply larger bias potentials in the lower-density LJ systems. At $n=0.012$, the phase transition is detected while $\alpha$ is of order of 100. At still lower density, for example, $n=0.01$, it is very difficult (if not impossible) to observe the transition using direct MD simulations. However, with our method we can still easily detect the gas/liquid transition. Fig.(2) shows the results for $n=0.008$ and $N=1000$. The distribution $P\left(S_{2}\right)$ is flatter and broader in the biased simulation, indicating that the system visits a larger conformational 
space. The lower panel of Fig.(2) shows the reproduced free energy profiles from the distribution of the biased simulation $\left(\alpha \approx 10^{6}\right)$. It greatly agrees with that from a non-biased simulation in the region where the direct MD is possible. The inset of Fig.(2) shows the distribution of samples in the $\left(V, S_{2}\right)$ space. The shown dense region $\left(S_{2}>-300\right)$ which corresponding to the gas phase is biased due to its higher distributed density of samples. The lower-density region shown in the inset $\left(S_{2}<-300\right)$ corresponds to the transition region ( the liquid phase which $S_{2}$ is far smaller -300 does not shown) was not biased. From the obtained distribution, we know $S_{2}$ is actually a good reaction coordinate, the difference between the rebuilt and biased $\log _{10} P\left(S_{2}\right)$, shown in the higher panel of Fig.(2), corresponds the profile of the applied bias potential (with a factor $k_{B} T \ln 10$ ).

To summarize, we have expanded the hyperdynamics method to more general cases by inhomogeneously compressing time and conformational space. Our approach directly generates an explicit general method to design the bias potential. In simple systems, a few functionals of the pair correlation function provide a good approximation of the density field for identifying the important conformations and for constructing the bias potential without prior knowledge of the conformational space. The method is expected to be applicable in more complex fluids where even more collective variables might be needed.

This work was supported by the US DOE under contract W-7405-ENG-36 with LDRD No. LA-UR-06-2794.

\section{Zhou et al, Fig.1}

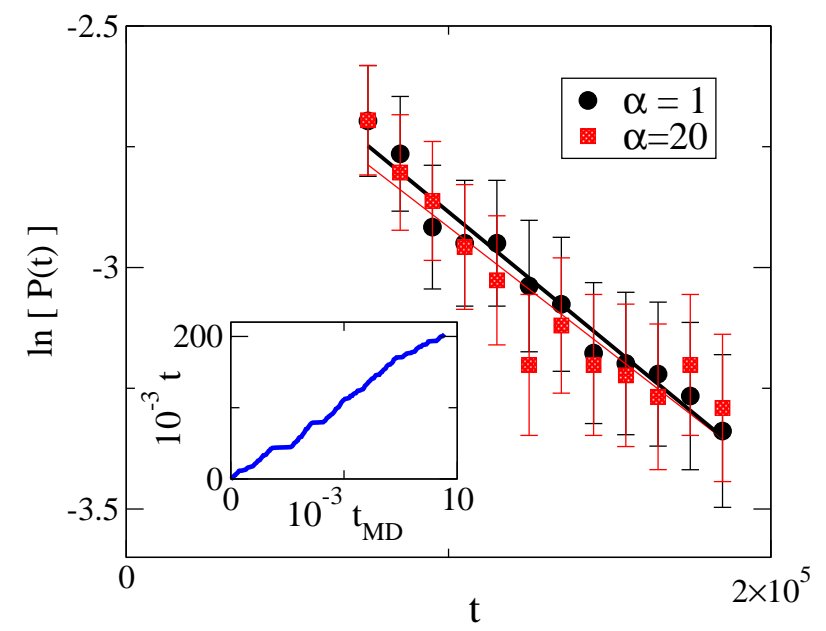

FIG. 1: (Color online). The distribution of transition time of the gas phase in direct MD $(\alpha=1)$ and biased MD $(\alpha=20)$ simulations. The inverse slope of the least-squares fit to the points (solid line) gives the lifetime of the gas phase. $n=$ 0.014, $N=400$ and $T=0.613$. Inset: the time boost in a typical biased MD simulation is shown.
X. Zhou et al, Fig.2

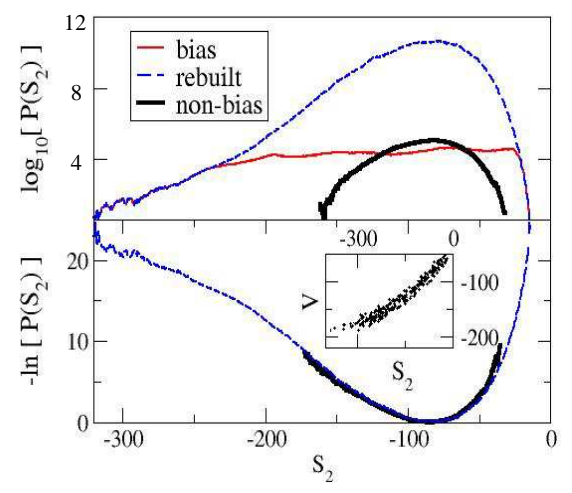

FIG. 2: (Color online). Top: the distributions of two body entropy $S_{2}$ from non-biased and biased simulations. The rebuilt distribution of the bias simulation is also shown. Here, $n=0.008, N=1000$ and $T=0.613$. Bottom: the free energy profiles from the non-biased and biased simulations are compared. The inset shows the simulated samples in the $\left(S_{2}, V\right)$ space. The observed liquid phase does not show here. 
[1] A. B. Bortz, M. H. Kalos, and J. L. Lebowitz, J. Comp. Phys. 17, 10 (1975).

[2] P. G. Bolhuis, D. Chandler, C. Dellago, and P. L. Geissler, Ann. Rev. Phys. Chem. 53, 291 (2002).

[3] R. Olender, and R. Elber, J. Chem. Phys. 105, 9299 (1996); B. K. Dey, M. R. Janicki, and P. W. Ayers, J. Chem. Phys. 121, 6667 (2004).

[4] A. Laio, and M. Parrinello, Proc. Natl. Acad. Sci. USA 99, 12562 (2002).

[5] R. Elber, Curr. Opin. Struct. Bio. 15, 151 (2005).

[6] A. F. Voter, Phys. Rev. Lett. 78, 3908 (1997); A. F. Voter, J. Chem. Phys. 106, 4665 (1997).

[7] R. A. Miron, and K. A. Fichthorn, Phys. Rev. Lett. 93,
028301 (2004).

[8] J. A. Rahman, and J. C. Tully, J. Chem. Phys. 116, 8750 (2002).

[9] D. Hamelberg, T.-Y. Shen, and J. A. McCammon, J. Chem. Phys. 122, 241103 (2005).

[10] M. M. Steiner, P.-A. Genilloud, and J. W. Wilkins, Phys. Rev. B 57, 10236 (1998).

[11] H. S. Green, The Molecular Theory of Fluids (NorthHolland, Amsterdam, 1952); A. Baranyai, and D. J. Evans, Phys. Rev. A 40, 3817 (1989); P. V. Giaquinta, G. Giunta, and S. PrestipinoGiarritta, Phys. Rev. A 45, R6966 (1992). 